\title{
Femtosecond Laser Scribing of Mo Thin Film on Flexible Substrate Using Axicon Focused Beam
}

\author{
Xiaoming $\mathrm{Yu}^{1}$, Jianfeng $\mathrm{Ma}^{2}$ and Shuting Lei ${ }^{{ }^{*}}$ \\ ${ }^{1}$ Department of Industrial and Manufacturing Systems Engineering, Kansas State University, USA \\ ${ }^{2}$ Department of Aerospace \& Mechanical Engineering, Saint Louis University, USA \\ philipyu@ksu.edu,jma15@slu.edu, lei@ksu.edu
}

\begin{abstract}
Ablation experiments of Molybdenum (Mo) thin film on flexible polyimide (PI) substrate are conducted using femtosecond laser pulses focused by an axicon lens. The purpose is to assess the capability of axicon lens in producing narrow grooves and how robustness the scribing process is. We first obtain the damage threshold of Mo and PI and then characterize the spatial beam profile produced by the axicon lens through both theoretical calculations and experimental measurements. Then scribing experiments are performed with different pulse energy, scanning speed, and the distance between the axicon tip and the sample's surface. Optical microscope and atomic force microscope are used to examine the microgrooves from the experiments. It is shown that high quality narrow scribes can be produced with axicon focused beam which can tolerate large height fluctuations of moving flexible substrate expected in an industrial setting.
\end{abstract}

Keywords: Mo thin film, flexible substrate, fs laser, axicon lens, laser micromachining

\section{Introduction}

The multilayer $\mathrm{CuIn}_{\mathrm{x}} \mathrm{Ga}_{\mathrm{x}} \mathrm{Se}_{2 \mathrm{y}} \mathrm{S}_{\mathrm{y}}$ (CIGS) thin film solar cell technology has drawn considerable attention due to its unique advantages of low production cost and high photovoltaic energy conversion efficiency. CIGS has been recognized as the most efficient thin-film solar cell technology with conversion efficiency of $20.4 \%$ reached in 2013 in a research lab (Hall, 2013). The material cost of CIGS thin-film cells can be very inexpensive since it requires few raw materials. The manufacturing cost can be reduced with an efficient, scalable roll-to-roll process. The conventional scribing process for monolithically integrated CIGS modules in production lines typically involves three steps: laser patterning of the Mo back conductor (P1), followed by mechanical patterning of the absorption layer

*Contact author: lei@ksu.edu 
(P2) and finally laser patterning of the front conductor layer (P3) (Powalla et al., 2006). Currently there is a push for establishing all laser-scribing in thin-film solar panel production.

Q-switched DPSS (diode pumped solid state) lasers emitting at $1064 \mathrm{~nm}$ or $355 \mathrm{~nm}$ (third harmonic) has been commonly used in industry for P1 scribe (Bartlome et al., 2010). The P2 and P3 processes are typically performed by a frequency-doubled DPSS laser emitting at $532 \mathrm{~nm}$. The pulse duration is usually around several tens of nanoseconds. Despite the popularity of ns lasers in thin film solar cell production, thermal damage due to melting, recast and microcrack formation such as shown by Haas et al. (2008) limits the scribe quality and line width to $>20 \mu \mathrm{m}$. The problem is more severe for CIGS type cells on flexible metal/polymer substrate because of the extreme heat sensitivity of the materials (Bartlome et al., 2010). Excessive melting of CIGS and deposition of molybdenum on scribe walls were found to reduce the photovoltaic efficiency considerably (Hermann et al., 2006a).

To overcome the shortcomings of ns lasers, ultrafast lasers with ps and fs pulses have been used to scribe CIGS solar cells. It was reported that all P1, P2 and P3 scribing can be performed by ps lasers with desired selectivity of different layers (Račiukaitis et al., 2010; Huber et al., 2008; Gečys et al., 2014). However, thermal effect was shown to be a problem even at this pulse duration (1 10 ps): periodic melting of the molybdenum (Mo) layer and damage to the substrate (especially polyimide) was observed in P1 scribing, electrical shunts were created near scribe edge, and non-uniform ablation leads to residuals in the groove (Wehrmann et al., 2012; Zhao et al., 2014; Wang et al., 2014). Studies using ultrashort pulses of a few hundred femtoseconds have been conducted and non-thermal ablation with narrow line width was reported for thin film scribes (Zoppel et al., 2007; Webb and Osborne, 2009). In addition, a few comparative investigations demonstrated unambiguously the advantages of fs pulses over ns and ps pulses in reduced thermal effects, minimal interdiffusion between multi-layers, improved electrical performance, and narrow line width (Kim et al., 2009; Hermann et al., 2006b; Ruthe et al., 2005). Recent research in ultrafast laser scribing has extended to use of pulse shaping in both spatial and temporal domain. Square top-hat beam profile has shown advantages such as reduced damage at groove center, smooth scribe edge and insensitiveness to beam quality (Račiukaitis et al., 2011). Bursts of low energy pulses were found to reduce melt areas in the scribe zone (Gečys et al., 2011).

Despite the advantage femtosecond laser offers in scribing CIGS solar cells, it faces one serious limitation: small depth of field. For example, the Rayleigh length for a laser with wavelength of $1 \mu \mathrm{m}$ focused to a $10 \mu \mathrm{m}$ spot size (in diameter) is less than $0.1 \mathrm{~mm}$, which means consistent narrow line width during scribing is impossible to achieve when the height variation for a large moving flexible substrate is expected to be up to $3 \mathrm{~mm}$ (Tamhankar et al., 2009). In view of the fact that common beam shaping from Gaussian to flattop distribution renders an even shorter depth of field based on the study by Tamhankar et al. (2009), the purpose of this paper is to study fs laser scribing with axicon focused beam. Use of an axicon lens can generate an intense beam pattern which is referred to as a near-nondiffracting beam, i.e., a beam with much larger depth of focus in comparison to that generated by a conventional focusing lens. Although the goal is to achieve all three patterning processes (P1, P2, P3) with an single fs laser source, the focus of this study is on P1, i.e., scribing Mo thin film on a flexible polyimide (PI) substrate.

\section{Experimental Setup and Procedure}

The experimental setup is shown in Fig. 1. A Ti:Sapphire femtosecond laser system delivers pulses with full-width-at-half-maximum (FWHM) pulse duration of $60 \mathrm{fs}$, center wavelength of $800 \mathrm{~nm}$, maximum pulse energy of $4.3 \mathrm{~mJ}$, beam diameter $\left(1 / \mathrm{e}^{2}\right)$ of $10 \mathrm{~mm}$, and repetition rate of $1 \mathrm{kHz}$. The pulse energy is attenuated by a variable neutral density (ND) filter. The attenuated beam is focused by an axicon lens (Doric Lenses). This axicon lens is made from fused silica, and has a base angle of $25^{\circ}$ and a tip angle of $130^{\circ}$. The thin-film sample used in this study consists of a back contact layer 
(molybdenum, Mo) and a PI substrate. The thicknesses of the Mo layer and the PI layer are $\sim 220 \mathrm{~nm}$ and $25 \mu \mathrm{m}$, respectively. The Mo thin film was deposited on the PI substrate using electron beam evaporation. The sample is mounted on a motorized XYZ stage (Newport).

Laser scribing experiments are performed on the sample by scanning it across the focal spot. Scribing conditions include pulse energy, scanning speed, and the distance (d) between the axicon tip and the sample's surface. We first place the sample at a fixed distance of $9 \mathrm{~mm}$ from the axicon tip, scribe grooves with various combinations of pulse energy and scanning speed, and examine groove quality using optical and atomic force microscopes. Then we apply the optimal combination of energy and speed found above and scribe grooves at different axicon-sample distances. The corresponding experimental conditions are shown in Tables 1 and 2, and the results are shown in Sections 3.3 and 3.4, respectively. Before conducting the scribing experiments, laser damage threshold of Mo and PI are determined and axicon focused beam profile is characterized to assist in the selection of laser scribing conditions.

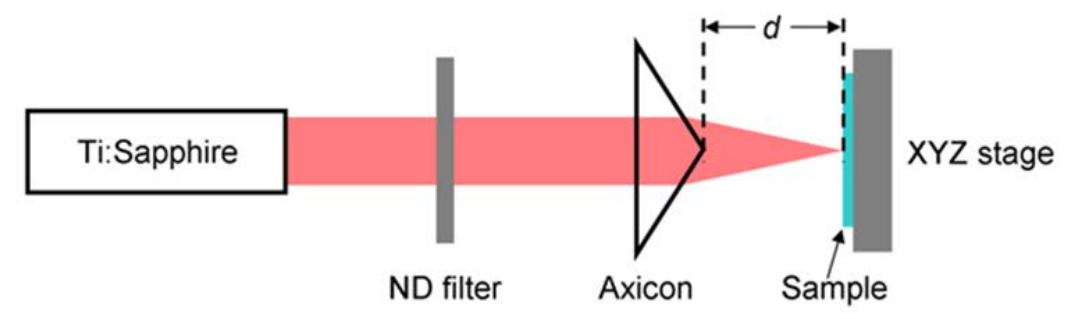

Fig. 1 Experimental setup

\begin{tabular}{|l|l|}
\hline Parameter & Value \\
\hline Pulse energy $(\mu \mathrm{J})$ & $10,15,20,25,30,35$ \\
\hline Laser scanning speed $(\mathrm{mm} / \mathrm{s})$ & $0.1,0.2, \ldots, 0.9,1.0$ \\
\hline Axicon tip to sample distance $(\mathrm{mm})$ & Fixed at $9 \mathrm{~mm}$ \\
\hline
\end{tabular}

Table 1. Experimental condition with fixed distance and different pulse energy and scanning speed.

\begin{tabular}{|l|l|}
\hline Parameter & Value \\
\hline Pulse energy $(\mu \mathrm{J})$ & Fixed at 15 \\
\hline Laser scanning speed $(\mathrm{mm} / \mathrm{s})$ & Fixed at 0.6 \\
\hline Axicon tip to sample distance $(\mathrm{mm})$ & $1,2,3, \ldots, 18 \mathrm{~mm}$ \\
\hline
\end{tabular}

Table 2. Experimental condition with fixed pulse energy and scanning speed at different distances.

\section{Results and Discussion}

\subsection{Single pulse damage threshold}

Damage threshold is a characteristic dependent on the wavelength, pulse width and type of material. It is ideally defined as the laser fluence at which irreversible damage occurs in the material by removing a monolayer of material. It is actually determined by visual examination, ablation depth 
measurement, plasma radiation monitoring etc. In this work the damage threshold is estimated by recording the diameter (D) of the ablated craters using SEM and then using the following relationship between the square of the crater diameter and the logarithm of the laser fluence (F) (Liu, 1982):

$D^{2}=2 w_{0}^{2} \ln \left(\frac{F}{F_{t h}}\right)$

where $\mathrm{w}_{0}$ and $\mathrm{F}_{\text {th }}$ are fitting variables, representing focal spot $1 / \mathrm{e}^{2}$ radius and damage threshold, respectively. A plot of the square of damage diameter, $\mathrm{D}^{2}$, against the logarithm of laser fluence is made to obtain both $\mathrm{w}_{0}$ (from the slope of the line) and $\mathrm{F}_{\text {th }}$ (from the extrapolation of $\mathrm{D}^{2}$ to zero value) as shown in Fig. 2. The Mo and PI damage threshold at $60 \mathrm{fs}$ were found to be $0.18 \mathrm{~J} / \mathrm{cm}^{2}$ and 0.75 $\mathrm{J} / \mathrm{cm}^{2}$, respectively. The Mo damage threshold slightly increases with increased pulse duration, which is consistent with the previous research of fs laser ablation of $\mathrm{Cu}$ and $\mathrm{Al}$ film and fs laser ablation of fused silica (Le Harzic et al., 2005; Perry et al., 1999). Since the damage threshold of PI is higher than that of Mo, it is possible to only remove the Mo layer while not damaging the PI substrate by choosing a laser fluence in between of these two thresholds. The optimal operating parameters (including pulse energy and scribing speed) are determined in the following scribing experiments.

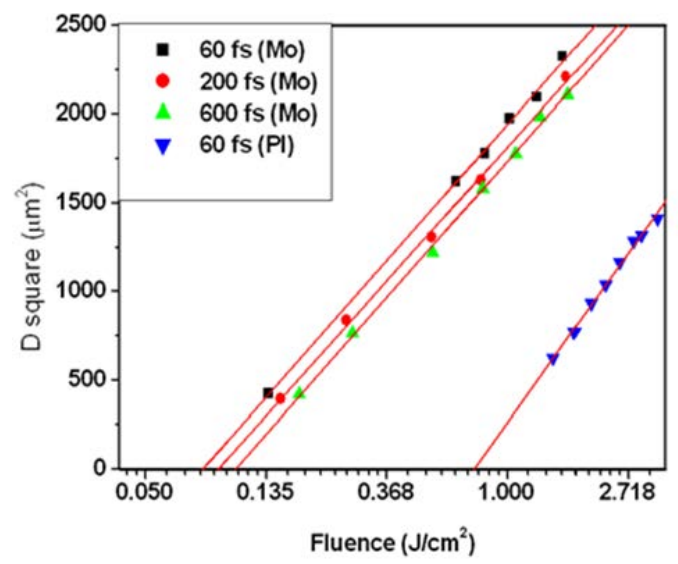

Fig. 2 Single pulse damage threshold of Mo and PI at various pulse durations

\subsection{Characterization of beam profile produced by axicon lens}

We first examine the beam profile after the axicon lens. Ideally, when an infinite plane wave is used as the input beam, an axicon lens with an infinite aperture) will generate an ideal Bessel beam, whose transverse intensity distribution, I(r), remains the same at arbitrary axial location, z, i.e., $\mathrm{I}(\mathrm{r}, \mathrm{z})=$ $\mathrm{I}(\mathrm{r}, 0)$ (Durnin, 1987). In our experiment, the input beam has a Gaussian profile, resulting in a BesselGauss beam after the axicon, and its intensity distribution is (Arlt and Dholakia, 2000).

$I(r, z)=I_{0} \cdot\left(4 \pi^{2} B^{2} \frac{z}{\lambda}\right) \cdot \exp \left[-\frac{2(B z)^{2}}{w_{0}^{2}}\right] \cdot J_{0}^{2}(k B r)$ 
where $\mathrm{I}(\mathrm{r}, \mathrm{z})$ is the intensity distribution after the axicon at transverse position (r) and axial position (z), $\mathrm{I}_{0}$ is the input beam intensity at $r=0, \lambda$ is the wavelength of the laser, $\mathrm{w}_{0}$ is the input beam $1 / \mathrm{e}^{2}$ radius, $\mathrm{J}_{0}$ is the zero order Bessel function, $\mathrm{k}$ is the wave number $(2 \pi / \lambda)$, and $\mathrm{B}$ is the beam deviation angle calculated by

$$
B=\arcsin (n \sin A)-A,
$$

where $\mathrm{n}$ and $\mathrm{A}$ are refractive index (1.45) and base angle $\left(25^{\circ}\right)$ of the axicon, respectively. Some of these variables are illustrated in Fig. 3 (a). It should be noted that in the derivation of the above equation, the paraxial assumption, $\sin B \sim B$, is used. For our axicon, $B=0.223$ rad and $\sin B=0.221$, which suggests that the paraxial assumption is valid.

In machining experiments with ultrashort pulses, laser fluence (F) instead of intensity (I) is the commonly used parameter, and these two are related by $\mathrm{F}=\mathrm{I} \tau$, where $\tau$ is the FWHM pulse duration. The calculated fluence distribution with $10 \mu \mathrm{J}$ pulse energy is shown in Fig. 3(d). Fig. 3(b) shows the fluence along the axial ( $\mathrm{z}$ ) axis at $\mathrm{r}=0$, whereas Fig. 3(c) shows the transverse distribution at $\mathrm{z}=11 \mathrm{~mm}$, where the on-axis fluence reaches the maximum.

From Fig. 3(d) we can see that the central spot size $(2.7 \mu \mathrm{m}$, measured between the first zeros) remains similar along the z-axis. Fig. 4 shows a comparison between the calculated focus profile (Fig. $3(\mathrm{a}))$ and the image of the focal spot seen from a CCD camera at $\sim 3 \mathrm{~mm}$ from the tip of the axicon lens. The measured center spot diameter is about $2.5 \mu \mathrm{m}$, which is similar to the calculated value.

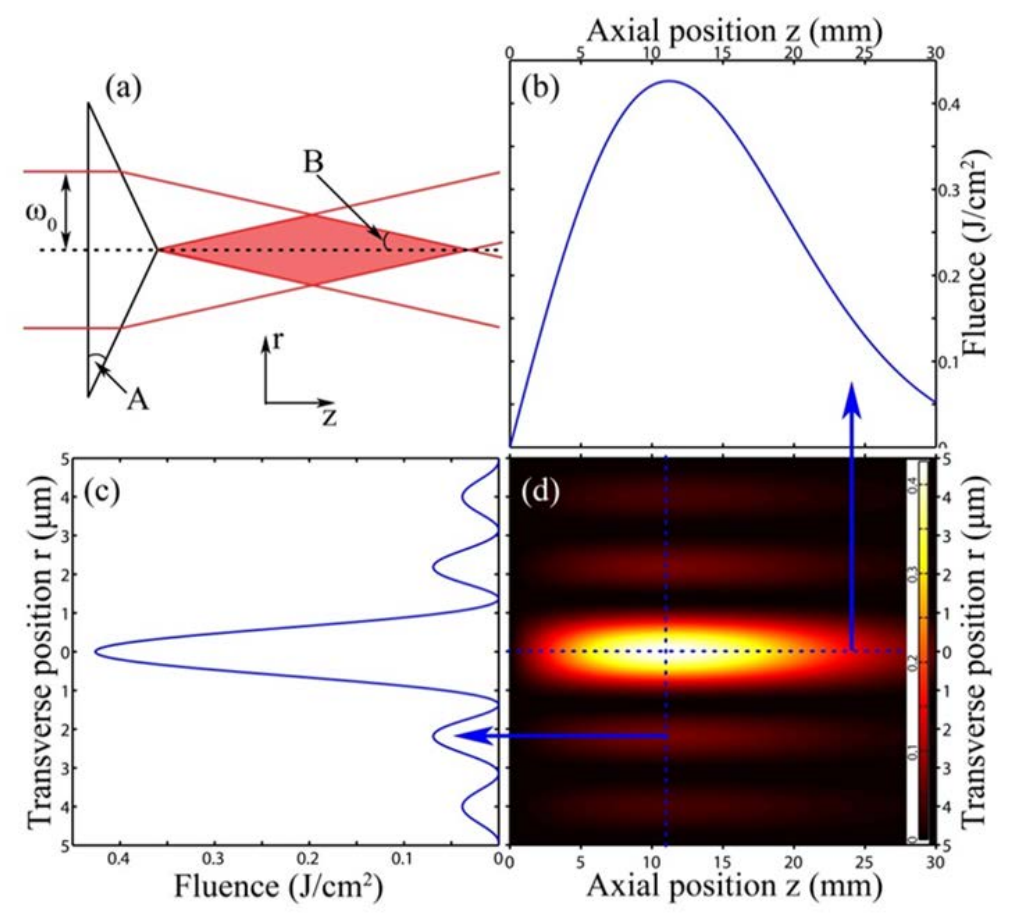

Fig. 3 (a) Schematic of the laser beam focused by an axicon with a base angle (A) of 25 deg. (b, c) Calculated fluence distribution along the axial and transverse direction, respectively. (d) 2-D plot of fluence distribution. Color bar represents fluence $\left(\mathrm{J} / \mathrm{cm}^{2}\right)$. Pulse energy is $10 \mu \mathrm{J}$. Please note that in $(\mathrm{d})$ the unit of the axial position $\mathrm{z}$ is $\mathrm{mm}$. 

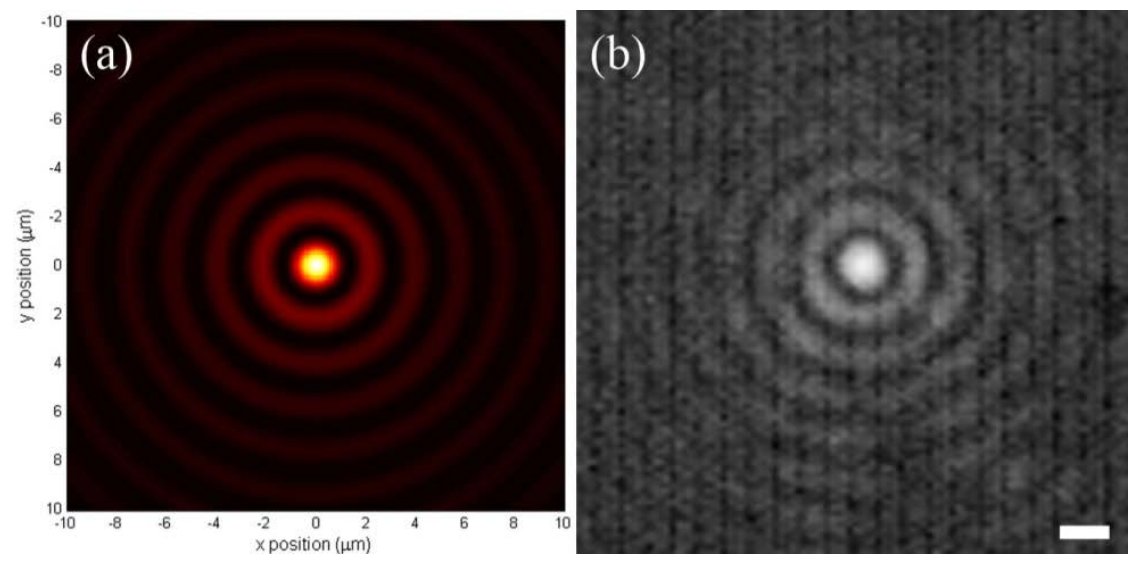

Fig. 4 Comparison of (a) calculated and (b) measured focal spot profile at $3 \mathrm{~mm}$ from the axicon tip. The scale bar in (b) is $2 \mu \mathrm{m}$. Both (a) and (b) are on the same spatial scale.

\subsection{Mo film scribing at fixed axicon-sample distance}

As can be seen in Fig. 3(b), the on-axis fluence reaches maximum when the axicon-sample distance is $11 \mathrm{~mm}$. However, it is found from our preliminary results (not shown here) that the maximum ablation effect occurs when this distance is around $9 \mathrm{~mm}$ instead of the predicted $11 \mathrm{~mm}$, perhaps this is because the spatial profile of our laser beam is not an ideal Gaussian shape. Therefore, in the following experiments in order to identify other parameters, such as the optimal pulse energy and scanning speed, we first place the sample at $9 \mathrm{~mm}$ from the axicon tip, and scribe grooves with different pulse energy and speeds.
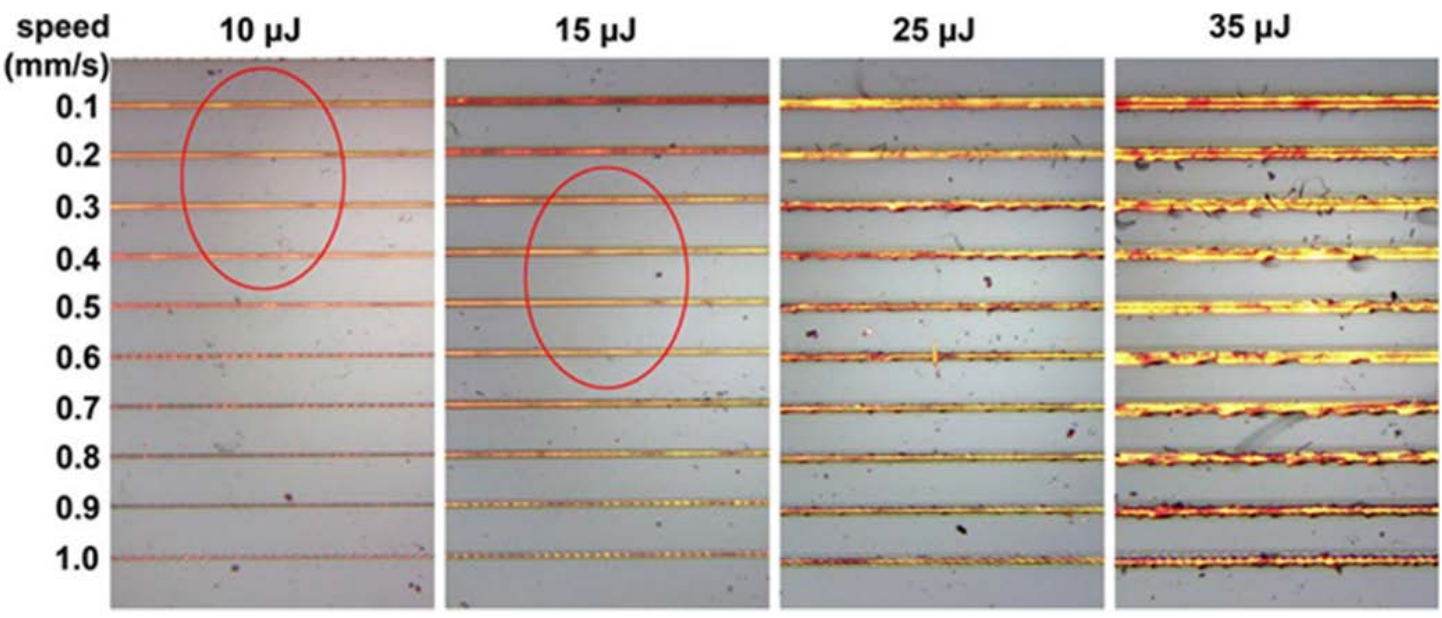

Fig. 5 Optic images of microgrooves fabricated by different speeds and laser power. The sample is moving transversely at a distance of $9 \mathrm{~mm}$ from the axicon tip. Red circles indicate the grooves with good quality. 
Figure 5 shows the optical images of narrow grooves written on the surface of Mo film with the axicon at different speeds and laser power and at a fixed axicon-sample distance of $9 \mathrm{~mm}$. These images are taken with both front and back illumination. Unprocessed area (between two adjacent lines) shows a white color, whereas written grooves show a yellowish color, indicating that the Mo film is completely or partially removed. As will be confirmed with AFM images (shown below), grooves with completely removed Mo film will show a brighter color compared to those with partially removed coating. Based on these optical imagers, we characterize good-quality grooves as those with bright colors and uniform, narrow widths. From Fig. 5 we can see that, at low pulse energy, the Mo film can only be removed at speeds of $<0.5 \mathrm{~mm} / \mathrm{s}$. At higher speeds, the effective energy deposition is less and only a part of the Mo layer is removed. At high energy (e.g. $35 \mu \mathrm{J})$, narrow black lines can be seen in the center of each groove at low speeds $(0.1 \sim 0.3 \mathrm{~mm} / \mathrm{s})$, indicating that the substrate is also damaged (confirmed with AFM imaging, results not shown). The line width is larger and the line edge is rougher compared to low power cases. This is perhaps because the rings of the Bessel beam also cause damage. It appears that good quality grooves (indicated by red circles in Fig. 5) can be achieved with either low energy, low speed $(10 \mu \mathrm{J}, 0.1-0.4 \mathrm{~mm} / \mathrm{s})$ or high energy, high speed $(15 \mu \mathrm{J}, 0.3-0.6$ $\mathrm{mm} / \mathrm{s})$. At high speed $(\sim 1 \mathrm{~mm} / \mathrm{s})$ there is an oscillation at the groove edges, perhaps due to a resonance in the motorized stages. More definitive observations can be made in the AFM images shown in Fig. 6 for some selected combinations of laser power and scribing speeds.

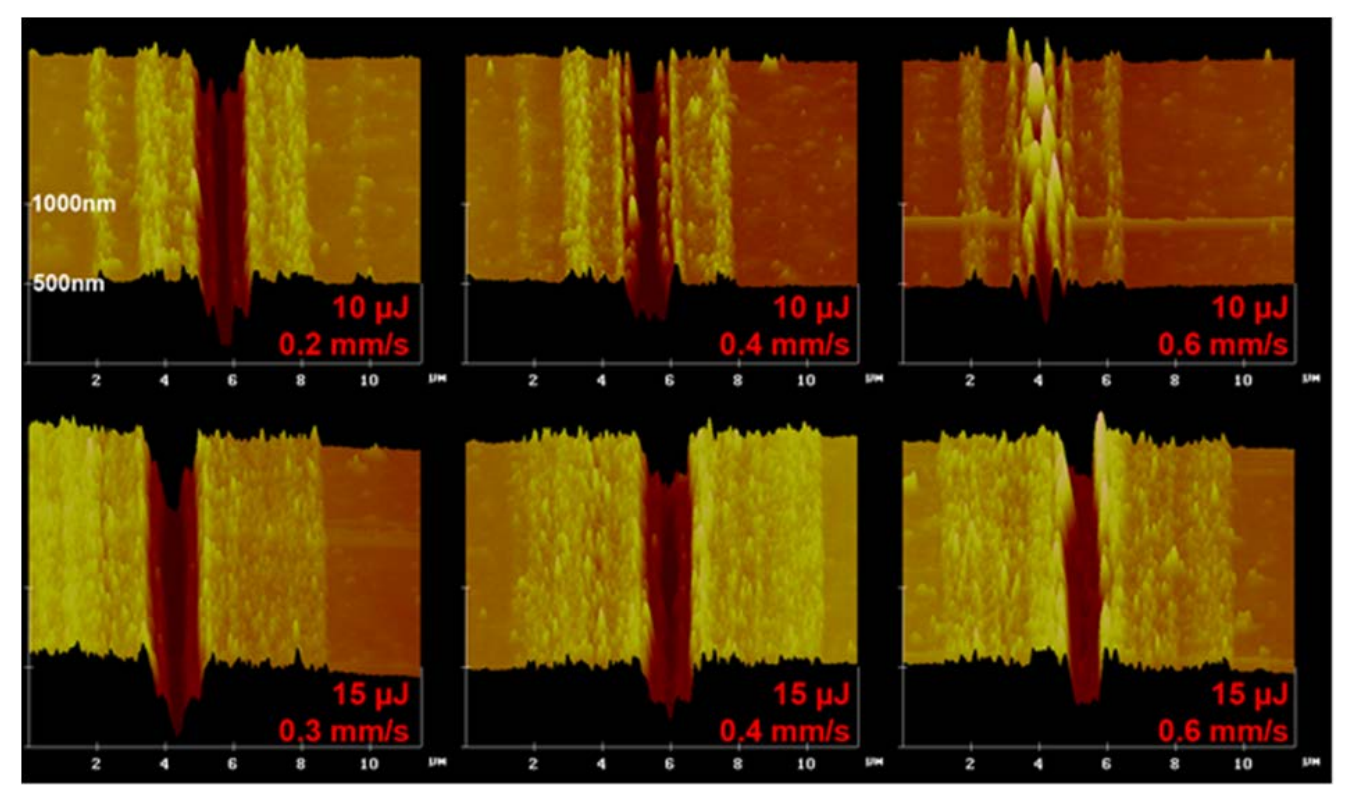

Fig. 6 AFM images of microgrooves fabricated at different scanning speeds and pulse energy. The distance from the sample surface to the axicon tip is fixed at $9 \mathrm{~mm}$.

These 3D AFM images of the microgrooves show clearly the groove profile as well as the surrounding areas. At the energy of $10 \mu \mathrm{J}$, the Mo film is removed at the speed of 0.2 and $0.4 \mathrm{~mm} / \mathrm{s}$, but not at $0.6 \mathrm{~mm} / \mathrm{s}$, matching the optical images. Substrate is damaged at $0.2 \mathrm{~mm} / \mathrm{s}$ as indicated by the deep narrow wedge extending downward from the groove bottom. Although the groove has a relatively flat bottom at $0.4 \mathrm{~mm} / \mathrm{s}$, the groove walls are lined with little bumps. As the power is increase slightly to $15 \mu \mathrm{J}$, cleaner grooves are obtained. With the speed increasing from 0.3 to 0.6 
$\mathrm{mm} / \mathrm{s}$, groove width decreases and damage to the substrate also diminishes. A nice groove is reached at $0.6 \mathrm{~mm} / \mathrm{s}$, characterized by flat bottom and straight walls. The groove width is about $2 \mu \mathrm{m}$ or less, which is expected because of the central spot size of the beam is about $2.5 \mu \mathrm{m}$. It is noted that a HAZ surrounds each groove on both sides and may span a few microns, which may be caused by the ring structures around the central spot. Further results (shown below) support such speculation.

It should also be noted that the optimal combination of pulse energy and scanning speed applies to our specific sample (Mo) and thickness $(220 \mathrm{~nm})$, and may differ for another material or thickness. However, as mentioned in Section 3 and will be demonstrated in the following section, the long focal range is a universal feature of a Bessel beam. Once the optimal combination is found in a similar way as presented in this section, order-of-magnitude improvement of focal range is expected.

\subsection{Mo film scribing at different axicon-sample distances}

With fixed pulse energy of $15 \mu \mathrm{J}$ and scanning speed of $0.6 \mathrm{~mm} / \mathrm{s}$, lines are written on the sample surface at different positions from the axicon tip (ranging from 1 to $18 \mathrm{~mm}$ for this condition), as shown in Fig. 7. No damage is observed when the distance is $1 \mathrm{~mm}$. From these images, we can see that when the distance is either small $(2-4 \mathrm{~mm})$ or large $(16-18 \mathrm{~mm})$, the Mo film is only partially removed (indicated by the $<200 \mathrm{~nm}$ depth), and the groove shape shows a high variability along the groove direction. In the middle portion of the distance $(6-13 \mathrm{~mm})$, the whole Mo layer is removed (indicated by the grooves' depth of $200 \mathrm{~nm}$ and their flat bottoms). In some locations the PI substrate is also damaged (shown in Fig. 8), and the width also shows variability. We attribute these to the fluctuation of pulse energy, non-uniformity of material and oscillation of the motorized stage. We can see that the groove depth and width remain similar within this distance range from 6 to $13 \mathrm{~mm}$, resulting in an effective range of $7 \mathrm{~mm}$, which is significantly larger than that for an ordinary lens or microscope objective for fabricating such narrow grooves with width of $2 \mu \mathrm{m}$. As an example, for a microscope objective of $\mathrm{NA}=0.5$ and laser wavelength of $800 \mathrm{~nm}$, the focal spot size is about $2 \mu \mathrm{m}$, and the Rayleigh range is $\mathrm{Z}_{\mathrm{R}}=\pi \omega_{0}{ }^{2} / \lambda=4 \mu \mathrm{m}$, resulting in a confocal parameter of only $8 \mu \mathrm{m}$, which should be compared to the axicon effective range of $7 \mathrm{~mm}$. Hence, by means of an axicon, the effective range for acceptable groove quality is about three orders of magnitude larger. The AFM images reveal that within this range, both the groove quality and width are consistent. This proves that axicon lens is a robust choice for consistent scribing quality when fluctuations in sample height are high.

A notable feature in Fig. 7 is the appearance of two pair of distinct lines with bump structures parallel to the central grooves, as can be clearly seen when the axicon-sample distance is $3 \mathrm{~mm}$ and 17 $\mathrm{mm}$ (indicated by Arrows 1), and $5 \mathrm{~mm}$ and $14 \mathrm{~mm}$ (indicated by Arrows 2). The locations of these two lines, $\sim 2 \mu \mathrm{m}$ and $\sim 4 \mu \mathrm{m}$ to the central groove, respectively, coincide with the positions of the first and second rings surrounding the central spot in the Bessel-Gauss beam, as can be seen from Fig. 3(c). Therefore we believe that these bump structures are caused by the ring structures in the beam. Moreover, the damage areas in each side of the central groove are also due to these ring structures. 


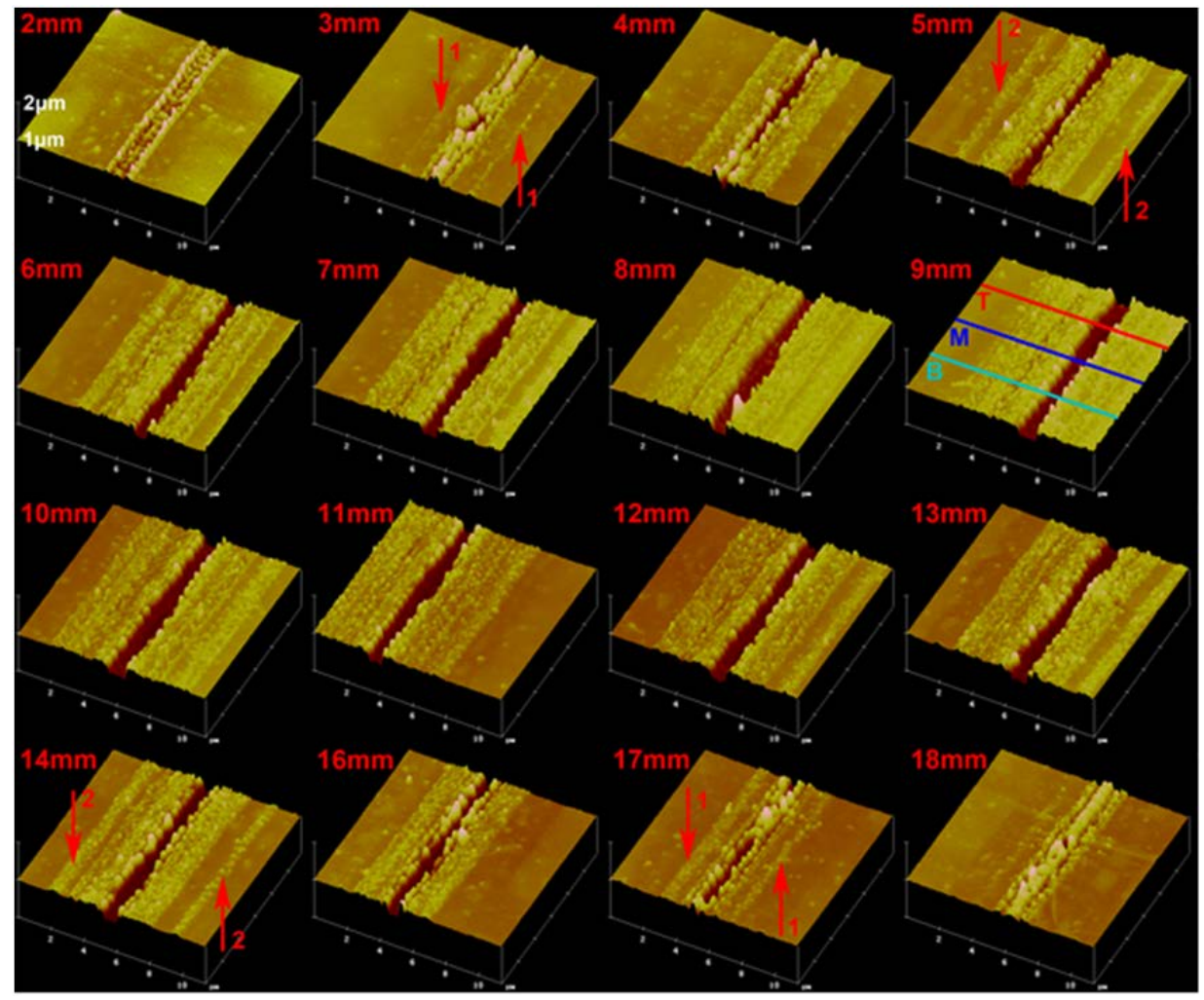

Fig. 7 AFM images of microgrooves fabricated at different distances between the sample surface and the axicon tip at the scanning speed of $0.6 \mathrm{~mm} / \mathrm{s}$ and pulse energy of $15 \mu \mathrm{J}$. Arrows indicate two pair of lines (numbered 1 and 2) with bump structures. In the $9 \mathrm{~mm}$ figure, T (Top), M (Middle) and B (Bottom) are three positions where cross sections are extracted. Cross sections at similar positions are also extracted in the $12 \mathrm{~mm}$ and $14 \mathrm{~mm}$ figures. No surface modification is observed for $1 \mathrm{~mm}$, and the image for $15 \mathrm{~mm}$ is similar to that for $14 \mathrm{~mm}$. Thus these two images are not shown.

Cross sections are extracted from Fig. 7 for the $9 \mathrm{~mm}, 12 \mathrm{~mm}$ and $14 \mathrm{~mm}$ figures, at three positions $\mathrm{T}$ (Top), $\mathrm{M}$ (Middle) and B (Bottom) in each figure, and are shown in Fig. 8. At $9 \mathrm{~mm}$, where the ablation effect is found to be maximal, PI substrate is clearly damaged at some location (the red Top curve). Better quality grooves with more uniform widths and reduced substrate damage are observed at $12 \mathrm{~mm}$. At $14 \mathrm{~mm}$, while the substrate damage is small, there is a larger width variation compared to the $12 \mathrm{~mm}$ case. More quantitative analysis is needed in the future to characterize the quality of each groove in terms of width, depth, heat affected zone (HAZ), electric insulation, etc. 

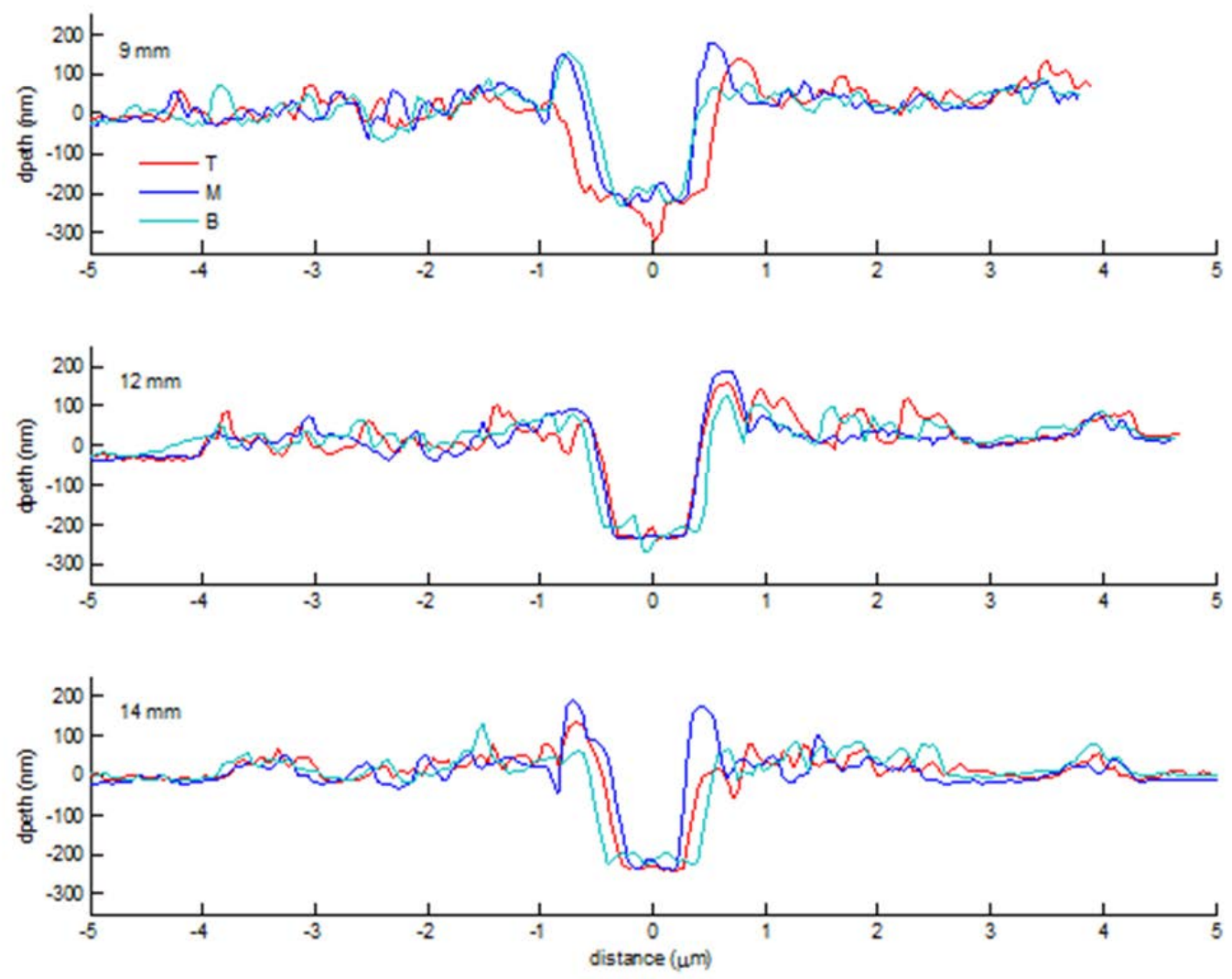

Fig. 8 Cross sections extracted from Fig. 7 along three positions indicated by T (Top), M (Middle) and B (Bottom) at $\mathrm{d}=9 \mathrm{~mm}, 12 \mathrm{~mm}$ and $14 \mathrm{~mm}$.

\section{Conclusions}

The large difference in the laser damage threshold between Mo and PI makes it possible for highly selective removal of Mo thin film on PI substrate. Use of a single axicon lens provides a simple and yet effective way of generating a Bessel like laser beam, which is characterized by a long focal length and small central spot size. The central spot of the Bessel beam has been proven to be a robust option for micromachining as shown in Mo film scribing, because it can provide fairly consistent groove quality while tolerating several millimeters of height fluctuations. Such results can be achieved with different combinations of laser power and scribing speeds. Future work involves quantitative characterization of groove quality, minimizing heat affected zone, and optimizing operating conditions. 


\section{Acknowledgments}

Financial support of this work by the National Science Foundation under Grant No. CMMI1131627 is gratefully acknowledged.

\section{References}

Arlt $\mathrm{J}$ and Dholakia K. Generation of high-order Bessel beams by use of an axicon. Optics Communications, 2000, 177: 297-301.

Bartlome R, Strahm B, Sinquin Y, Feltrin A and Ballif C. Laser applications in thin-film photovoltaics. Applied Physics B: Lasers and Optics, 2010, 100(2): 427-436.

Durnin J. Exact solutions for nondiffracting beams. I. The scalar theory. Journal of the Optical Society of America A, 1987, 4: 651-654.

Gečys P, Markauskas E, Dudutis J and Račiukaitis G. Interaction of ultra-short laser pulses with CIGS and CZTSe thin films. Applied Physics A., 2014, 114: 231-241.

Gečys P, Račiukaitis G, Miltenis E, Braun A and Ragnow S. Scribing of thin-film solar cells with picoseconds laser pulses. Physics Procedia, 2011, 12: 141-148. 21.

Hall M. EMPA announces 20.4\% efficient thin film CIGS-on-polymer cell. PV Magazine, 2014, 1:

Haas S, Schöpe G, Zahren C and Stiebig H. Analysis of the laser ablation processes for thin-film silicon solar cells. Applied Physics A: Materials Science \& Processing, 2008, 92: 755-759.

Hermann J, Benfarah M, Bruneau S, Axente E, Coustillier G, Itina T, Guillemoles JF and Alloncle P. Comparative investigation of solar cell thin film processing using nanosecond and femtosecond lasers. Journal of Physics D: Applied Physics, 2006a, 39(3): 453-460.

Hermann J, Benfarah M, Coustillier G, Bruneau S, Axente E, Guillemoles JF, Sentis M, Alloncle P and Itina T. Selective ablation of thin films with short and ultrashort laser pulses. Applied Surface Science, 2006b, 252: 4814-4818.

Huber HP, Herrnberger F, Kery S and Zoppel S. Selective structuring of thin-film solar cells by ultrafast laser ablation. Proceedings of SPIE, 2008, 6881: 688117-1-688117-10.

Kim TW, Pahk HJ, Park HK, Hwang DJ and Grigoropoulos CP. Comparison of multilayer laser scribing of thin film solar cells with femto, pico and nanosecond pulse durations. Proceedings of SPIE, 2009, 7409: 74090A-1-74090A-10.

Le Harzic R, Breitling D, Weikert M, Sommer S, Fohl C, Valette S, Donnet C, Audouard E and Dausinger F. Pulse width and energy influence on laser micromachining of metals in a range of $100 \mathrm{fs}$ to 5 ps. Applied Surface Science, 2005, 249: 322-331.

Liu JM. Simple technique for measurements of pulsed Gaussian-beam spot sizes. Optics Letters, 1982, 7: 196-198.

Perry MD, Stuart BC, Banks PS, Feit MD, Yanovsky V and Rubenchik AM. Ultrashort-pulse laser machining of dielectric materials. Journal of Applied Physics, 1999, 85(9): 6803-6810.

Powalla M, Cemernjak M, Eberhardt J, Kessler F, Kniese R, Mohring HD and Dimmler B. Largearea CIGS modules: Pilot line production and new developments. Solar Energy Materials and Solar Cells, 2006, 90(18-19): 3158-3164.

Račiukaitis G, Stankevičius E, Gečys P, Gedvilas M, Bischoff C, Jäger E, Umhofer U and Välklein F. Laser processing by using diffractive optical laser beam shaping technique. Journal of Laser Micro/nanoengineering, 2011, 6(1): 37-43.

Ruthe D, Zimmer K and Hoche T. Etching of CuInSe2 thin films-comparison of femtosecond and picosecond laser ablation. Applied Surface Science, 2005, 247: 447-452. 
Tamhankar A, Bovatsek J, Guadano G and Patel R. Advantages of using DPSS nanosecond laser with a Gaussian beam shape for scribing thin film photovoltaic panels. The Proceedings of the 2009 ICALEO Congress, Laser Microprocessing Conference, 2009, pp. 1251-1259.

Wang X, Ehrhardt M, Lorenz P, Scheit C, Ragnow S, Ni XW and Zimmer K. The influence of the laser parameter on the electrical shunt resistance of scribed $\mathrm{Cu}(\mathrm{InGa}) \mathrm{Se} 2$ solar cells by nested circular laser scribing technique. Applied Surface Science, 2014, 302: 194-197.

Webb A and Osborne M. Investigation of Laser Scribing Techniques to Improve Thin Film Solar Cell Manufacturability and Performance. Conference Record of the IEEE Photovoltaic Specialists Conference, the 34th IEEE Photovoltaic Specialists Conference, 2009, pp. 002480-002485.

Wehrmann A, Puttnins S, Hartmann L, Ehrhardt M, Lorenz P and Zimmer K. Analysis of laser scribes at CIGS thin film solar cells by localized electrical and optical measurements. Optics \& Laser Technology, 2012, 44: 1753-1757.

Zhao X, Cao Y, Nian Q, Shin YC and Cheng G. Precise selective scribing of thin-film solar cells by a picoseconds laser. Applied Physics A, 2014, 116: 671-681.

Zoppel S, Huber H and Reider GA. Selective ablation of thin Mo and TCO films with femtosecond laser pulses for structuring thin film solar cells. Applied Physics A, 2007, 89: 161-163. 